\title{
Les Pratiques De Veille Chez Le Haut Manager Marocain : Quelles Echelles De Mesure?
}

\author{
Hamdoune Amine \\ Doctorant à l'Université Abdelmalek Essaâdi, FSJES de Tanger, Morocco \\ CEDoc « Economie et Gestion » \\ Groupe de Recherche « Management et Système d'information » \\ Derkaoui Moulay Hicham
}

Doctorant à l'Université Mohammed V de Rabat, FSJES de Salé, Morocco CEDoc «Droit Comparé, Economie Appliquée et Développement Durable»

Formation «Economie Appliquée »

Equipe de Recherche « Monnaie, Banque et Marchés Financiers »

Doi: 10.19044/esj.2017.v13n31p394 URL:http://dx.doi.org/10.19044/esj.2017.v13n31p394

\begin{abstract}
In order to explain the factors influencing the use of business intelligence by senior managers, this paper seeks to sift through all the measuring instruments that have been used, in previous work, to operationalize the variables and the dimensions forming our theoretical construct. Based on Churchill's (1979) paradigm, which proposes a systematic procedure, this choice of items will result in the administration of a questionnaire. Also, it is aimed at testing our conceptual model in the context of Moroccan industrial enterprises.
\end{abstract}

Keywords: Scales measure, Environmental scanning, Behavior of use, Perceived Strategic Uncertainty, Perceived Source Accessibility, Perceived Source Quality

Résumé

Dans l'objectif d'expliquer les facteurs influençant l'utilisation de la veille stratégique par les hauts managers, ce papier cherche à passer au crible l'ensemble des instruments de mesure ayant été utilisés, lors des travaux antérieurs, pour opérationnaliser les variables et les dimensions formant notre construit théorique. Appuyé sur le paradigme de Churchill (1979) qui propose une procédure systématique, ce choix des items aboutira à l'administration d'un questionnaire qui vise le test de notre modèle conceptuel dans le contexte des entreprises industrielles marocaines. 
Mots-clés: Echelles de mesure, Veille stratégique, Incertitude stratégique perçue, Accessibilité perçue de l'information, Qualité perçue de l'information

\section{INTRODUCTION}

Dans l'objectif d'expliquer les facteurs influençant l'utilisation de la veille stratégique par les hauts managers, ce travail de recherche nous permettra de passer au crible l'ensemble des échelles de mesure qui vont opérationnaliser les variables formant notre modèle de recherche (Figure N¹). Appuyé sur le paradigme de Churchill (1979) qui propose une procédure systématique, ce choix des items aboutira à l'administration d'un questionnaire qui vise le test de notre construit théorique dans le contexte des entreprises industrielles marocaines.

L'objectif assigné à un instrument de mesure; tel que le questionnaire; est de tendre vers l'obtention d'une mesure parfaite du phénomène étudié (vraie valeur). Comme c'est le cas de notre recherche, cette quête est difficile puisque l'étude porte sur des perceptions subjectives. Ce paradigme s'inscrit dans la théorie de la mesure qui vise à tester la qualité des instruments de mesure. Il est fondé sur la notion (vraie valeur) formalisée de la manière suivante (Evrard et al., 1993) : Mesure obtenue $=$ Vraie valeur + Erreur systématique + Erreur aléatoire.

Selon cette procédure rigoureuse qui se base sur une approche exploratoire, nous allons légitimer le choix de nos instruments de mesure sur la base d'un croisement d'une revue de littérature par rapport aux recherches antérieures (Théorique et empirique) ayant traité la problématique de l'utilisation de veille stratégique par les hauts managers d'une part, et les résultats d'une étude qualitative réalisée via des entretiens semi directifs avec 13 hauts managers exerçant dans le contexte industriel marocain d'une autre part. Un croisement qui sera focalisé sur chacune des variables de notre construit de recherche à savoir: l'incertitude stratégique perçue ; l'accessibilité perçue d'une source d'information; la qualité perçue d'une source d'information; la fréquence de veille ; le mode de veille. 


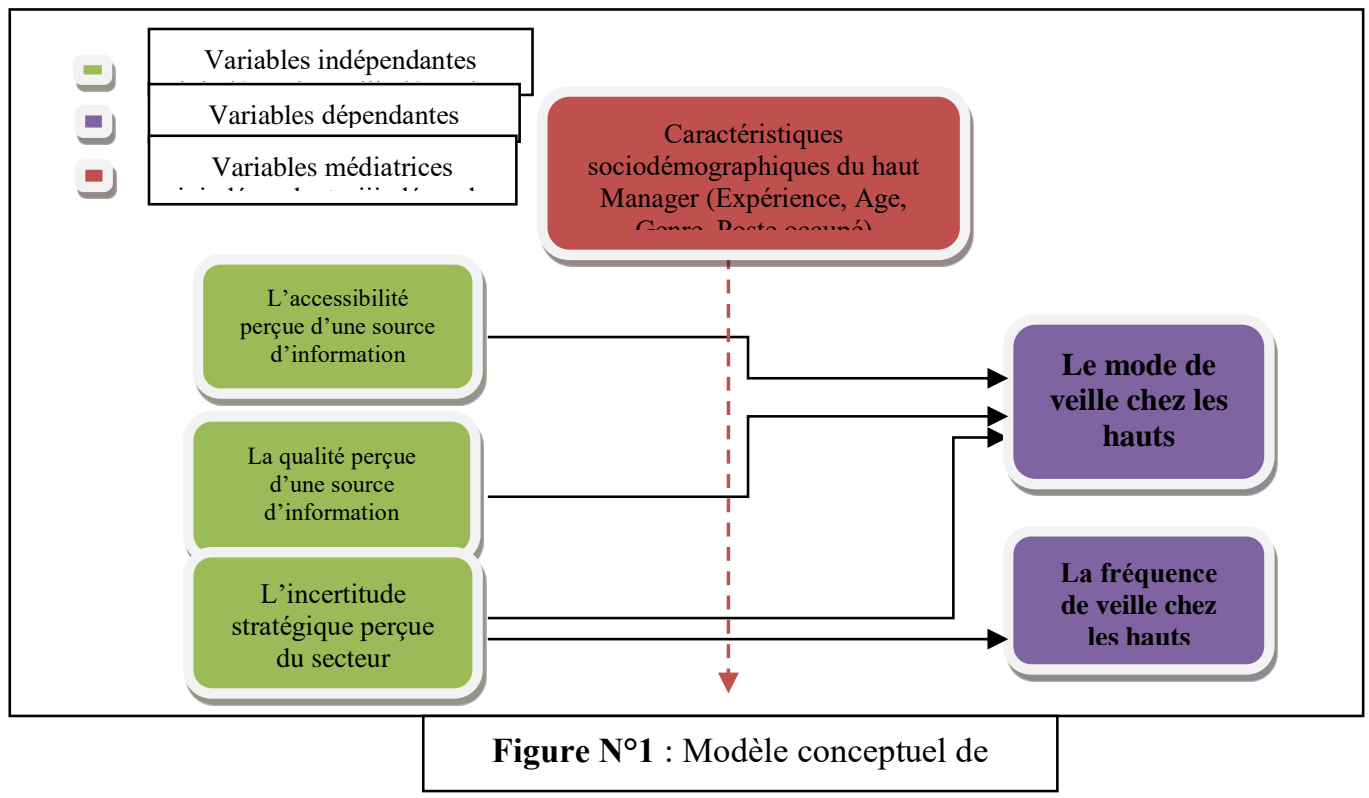

\section{La Veille Stratégique}

La symbiose avec l'environnement est l'une des raisons principales qui rendent l'écoute de l'environnement une condition indispensable pour la survie et le développement de l'entreprise. En effet, les auteurs en management stratégique mettent en avant la nécessité d'un processus d'écoute de l'environnement pour les entreprises pour collecter et analyser des informations qui n'ont pas seulement de conséquences immédiates apparentes sur les décisions mais qui permettraient également de détecter les menaces et les opportunités potentielles. Ces informations nécessitent un système d'information spécifique permettant de repérer les changements dès leurs premiers signes. Ce système d'information s'appelle la Veille Stratégique : "La VS est un système d'information d'aide à la décision, parmi ceux qui ont comme objectif de fournir des informations aux décideurs et de les assister dans leur processus de décision »(Reix, 2000) (Cité par Boulifa, 2008).

En dépit de la panoplie des définitions de la veille stratégique suggérées par les auteurs, nous optons dans le cadre de ce travail pour la définition d'Aguilar (1976) qui reste à notre sens la plus pertinente : «Scanning is the activity of acquiring information about events and relationships in a company's outside environment, the knowledge of which would assist top management in its task of charting the company's future course of action » (Cité par Daft et al., 1988). 
Il est très difficile de mesurer une activité de veille. Une difficulté liée principalement à l'absence d'un modèle organisationnel créant l'unanimité ainsi que le caractère souvent informel et spécial du processus de veille stratégique au sein de l'organisation (Aguilar 1967; Mintzberg, 1973). Il n'est pas seulement difficile d'explorer empiriquement cette pratique, même les hauts managers trouvent beaucoup de difficultés à décrire précisément leurs comportements de veille.

Cette difficulté en matière de mesure a poussé les chercheurs a essayé plusieurs méthodes et approches selon leurs contextes, basés partiellement sur des mesures adoptées par les anciens chercheurs et parfois sur de nouvelles mesures adaptées aux spécificités de leurs contextes de recherche. Hambrick (1982) a recommandé la mesure de l'utilisation de la veille stratégique à l'aide de plusieurs indicateurs soit la fréquence de veille, le niveau d'intérêt ou les heures consacrées à l'activité de veille. Bien que les deux premiers indicateurs ont reçu un large soutien au niveau de la littérature, l'indicateur basé sur le nombre d'heures consacrées à la veille était moins commode (Farh, Hoffman \& Hegarty, 1984; Cité par Daft et al., 1988).

Lors de nos entretiens semi-directifs avec les hauts managers de l'industrie pharmaceutique marocaine, on a pu constater chez nos interlocuteurs une bonne maitrise du concept sur le plan sémantique ainsi qu'une acceptation des items liés à la pratique de veille stratégique comme « la fréquence de veille» et «l'intérêt à la veille». Concernant l'item lié au "nombre d'heures consacrées à la veille », on a remarqué une très forte hésitation chez les hauts managers pour donner un nombre précis d'heures.

Extraits de verbatim illustrant notre propos:

Un haut manager (Responsable de production) a essayé d'expliquer ses pratiques de veille en utilisant des termes comme «intérêt et fréquence » et évite le « nombre d'heures consacrées à la veille ».: «[...] La veille stratégique au sens large du terme c'est l'ensemble des mécanismes, un ensemble de méthodes qui permettent au responsable d'être éveillé et regardant dans son domaine [...] on pratique de la veille en collectant fréquemment de l'information provenant de la concurrence et de la clientèle cible afin de prendre des décisions de plus en plus performantes [...] Je m'intéresse beaucoup plus à la concurrence [...] aaaaa, il m'est difficile de parler d'un nombre exact d'heures » Interviewé $n^{\circ} 11$.

Un autre haut manager (Directeur des RH) a exprimé ses pratiques de veille concentrées sur la concurrence et la clientèle en utilisant le terme "fréquentes" : "[...] veille stratégique!!!! C'est d'avoir un pas d'avance par rapport à tous mes concurrents... c'est un concept qui est vraiment difficile à réaliser [...] mes pratiques de veille sont plus fréquentes vis-à-vis de la clientèle et de la concurrence» Interviewé $\boldsymbol{n}^{\circ} \mathbf{3}$. 


\subsection{La Fréquence de Veille}

Considéré comme l'instrument le plus commode pour mesurer la pratique de veille stratégique, "la fréquence de veille" se réfère à la fréquence par laquelle les gestionnaires surveillent leurs macro et micro environnements.

En 1982 aux Etats Unis d'Amérique, Hambrick avec son travail intitulé "Environmental Scanning and Organizational Strategy», la fréquence de veille par rapport à chaque secteur (20 secteurs) de l'environnement a été mesurée à l'aide d'une échelle de Likert de sept (07) points. L'échelle de mesure de la fréquence de veille pour chaque secteur de l'environnement se situe entre extrêmement rare (1) et extrêmement fréquente (7). Les vingt (20) secteurs de l'environnement ont été choisis par Hambrick parmi quarante (40) secteurs environnementaux dégagés à partir d'une revue de la littérature et à l'aide d'un ensemble d'experts opérant dans le domaine industriel américain.

En 1988 aux Etats Unis d'Amérique, Daft et d'autres avec leur travail intitulé "Chief executive scanning, environmental characteristics, and company performance: an empirical study », La fréquence de veille par rapport à Six secteurs de l'environnement a été mesurée à l'aide d'une échelle de mesure similaire à celle utilisée par Hambrick en 1982 et Culnan 1984 et validée par Farh et d'autres en 1984. Cet instrument de mesure se base sur une échelle de Likert de cinq (05) points. L'échelle de mesure de la fréquence de veille pour chaque secteur de l'environnement se situe entre (chaque jour et moins d'une fois par an) passant par une fois par semaine, une fois par mois et plus qu'une fois par an.

Tableau No1. Instrument de mesure de la fréquence de veille

\begin{tabular}{|c|c|c|c|c|c|}
\hline & $\begin{array}{c}\text { chaque } \\
\text { jour }\end{array}$ & $\begin{array}{c}\text { chaque } \\
\text { semaine }\end{array}$ & $\begin{array}{c}\text { chaque } \\
\text { mois }\end{array}$ & $\begin{array}{c}\text { dius } \\
\text { d'une } \\
\text { fois par } \\
\text { an }\end{array}$ & $\begin{array}{c}\text { moins } \\
\text { d'une fois } \\
\text { par an }\end{array}$ \\
\hline Secteur de la concurrence & 1 & 2 & 3 & 4 & 5 \\
\hline Secteur de la clientèle & 1 & 2 & 3 & 4 & 5 \\
\hline $\begin{array}{c}\text { Environnement } \\
\text { technologique }\end{array}$ & 1 & 2 & 3 & 4 & 5 \\
\hline Environnement juridique & 1 & 2 & 3 & 4 & 5 \\
\hline $\begin{array}{c}\text { Environnement } \\
\text { économique }\end{array}$ & 1 & 2 & 3 & 4 & 5 \\
\hline $\begin{array}{c}\text { Environnement } \\
\text { socioculturel }\end{array}$ & 1 & 2 & 3 & 4 & 5 \\
\hline
\end{tabular}

Source : Daft et d'autres (1988)

En 1993 au Canada, Choo Wei Chun avec son travail intitulé «Environmental scanning: acquisition and the use of information by chief executive officers in the canadian telecommunications industry » et lors de 
son travail avec Auster Eyhel (1993) intitulé «Environmental scanning by CEO's in two Canadian industries », la fréquence de veille par rapport à Six secteurs de l'environnement a été mesurée à l'aide d'une échelle de mesure similaire à celle utilisée par Daft 1988, Hambrick en 1982 et Culnan 1984. Cet instrument de mesure se base sur une échelle de Likert de cinq (05) points. L'échelle de mesure de la fréquence de veille pour chaque secteur de l'environnement se situe entre (moins d'une fois par an et minimum une fois par jour) plus qu'une fois par an, minimum une fois par mois et minimum une fois par semaine.

Tableau $\mathrm{N}^{\circ}$ 2. Instrument de mesure de la fréquence de veille

\begin{tabular}{|c|c|c|c|c|c|}
\cline { 2 - 6 } & $\begin{array}{c}\text { moins d'une } \\
\text { fois par an }\end{array}$ & $\begin{array}{c}\text { plus d'une } \\
\text { fois par an }\end{array}$ & $\begin{array}{c}\text { Minimum } \\
\text { une fois par } \\
\text { mois }\end{array}$ & $\begin{array}{c}\text { Minimum } \\
\text { une fois par } \\
\text { semaine }\end{array}$ & $\begin{array}{c}\text { Minimum une } \\
\text { fois par jour }\end{array}$ \\
\hline $\begin{array}{c}\text { Secteur de la } \\
\text { concurrence }\end{array}$ & 1 & 2 & 3 & 4 & 5 \\
\hline Secteur de la clientèle & 1 & 2 & 3 & 4 & 5 \\
\hline $\begin{array}{c}\text { Environnement } \\
\text { technologique }\end{array}$ & 1 & 2 & 3 & 4 & 5 \\
\hline $\begin{array}{c}\text { l'environnement } \\
\text { juridique }\end{array}$ & 1 & 2 & 3 & 4 & 5 \\
\hline $\begin{array}{c}\text { l'environnement } \\
\text { économique }\end{array}$ & 1 & 2 & 3 & 4 & 5 \\
\hline $\begin{array}{c}\text { l'environnement } \\
\text { socioculturel }\end{array}$ & 1 & 2 & 3 & 4 & 5 \\
\hline
\end{tabular}

Source : Choo Wei Choo et E. Auster (1993)

En 1997, Detelin s. Elenkov dans son travail en Bulgarie : "strategic uncertainty and environmental scanning: the case for institutional influences on scanning behavior", La fréquence de veille par rapport à Six secteurs de l'environnement a été mesurée à l'aide d'une échelle de mesure similaire à celle utilisée par Hambrick en 1982 et Culnan 1984 et validée par Farh et d'autres en 1984. Cet instrument de mesure se base sur une échelle de Likert de sept (07) points. L'échelle de mesure de la fréquence de veille pour chaque secteur de l'environnement se situe entre (Une fois par an ou moins et une fois par jour ou plus).

En 2009 à Hong kong, Giri Jogaratnam dans son travail « Environmental Uncertainty and Scanning Behavior: An Assessment of Top-Level Hotel Executives ", La fréquence de veille par rapport à Six secteurs de l'environnement a été mesurée à l'aide d'une échelle de mesure similaire à celle utilisée par Ebrahimi 2000. Cet instrument de mesure se base sur une échelle de sept (07) points. L'échelle de mesure de la fréquence de veille pour chaque secteur de l'environnement se situe entre (Rarement (1) et très fréquemment (7)). 


\subsection{L'intérêt à La Veille}

Considéré comme l'un des instruments les plus commodes pour mesurer la pratique de veille stratégique, "l'intérêt à la veille "se réfère au degré d'intérêt accordé par les gestionnaires à leurs macro et micro environnements lors de la pratique de veille.

En 1993 au Canada, Choo Wei Chun avec son travail intitulé «Environmental scanning: acquisition and the use of information by chief executive officers in the canadian telecommunications industry » et lors de son travail avec Auster Eyhel (1993) intitulé «Environmental scanning by CEO's in two Canadian industries ", L'intérêt à la veille par rapport à six secteurs de l'environnement a été mesurée en proposant aux questionnés cinq (05) niveaux d'intérêt.

Tableau N³. Instrument de mesure de l'intérêt à la veille

\begin{tabular}{|c|c|c|c|c|c|}
\hline & $\begin{array}{c}\text { Je n'essaye } \\
\text { pas } \\
\text { généralement } \\
\text { d'être au } \\
\text { courant de ce } \\
\text { type } \\
\text { d'informations }\end{array}$ & $\begin{array}{c}\text { J'essaye d'être } \\
\text { au courant par } \\
\text { rapport aux } \\
\text { tendances et } \\
\text { événements } \\
\text { majeurs de ce } \\
\text { secteur de } \\
\text { l'environnement }\end{array}$ & $\begin{array}{c}\text { J'essaye } \\
\text { d'être au } \\
\text { courant en } \\
\text { détail par } \\
\text { rapport aux } \\
\text { tendances et } \\
\text { événements } \\
\text { majeurs de } \\
\text { ce secteur }\end{array}$ & $\begin{array}{c}\text { J'essaye } \\
\text { d'être au } \\
\text { courant des } \\
\text { détails de la } \\
\text { plupart des } \\
\text { tendances ou } \\
\text { événements } \\
\text { de ce secteur }\end{array}$ & $\begin{array}{c}\text { J'essaye } \\
\text { de } \\
\text { connaître } \\
\text { tous ce } \\
\text { que je dois } \\
\text { connaître } \\
\text { de ce } \\
\text { secteur }\end{array}$ \\
\hline $\begin{array}{c}\text { Secteur de la } \\
\text { concurrence }\end{array}$ & 1 & 2 & 3 & 4 & 5 \\
\hline $\begin{array}{c}\text { Secteur de la } \\
\text { clientèle }\end{array}$ & 1 & 2 & 3 & 4 & 5 \\
\hline $\begin{array}{c}\text { Environnement } \\
\text { technologique }\end{array}$ & 1 & 2 & 3 & 4 & 5 \\
\hline $\begin{array}{c}\text { l'environnement } \\
\text { juridique }\end{array}$ & 1 & 2 & 3 & 4 & 5 \\
\hline $\begin{array}{c}\text { l'environnement } \\
\text { économique }\end{array}$ & 1 & 2 & 3 & 4 & 5 \\
\hline $\begin{array}{c}\text { l'environnement } \\
\text { socioculturel }\end{array}$ & 1 & 2 & 3 & 4 \\
\hline
\end{tabular}

Source : Choo (1993)

En 2001 en Botswana, Zelealem T. Temtime dans son travail «Environmental scanning behavior of small and medium firms in developing economies: Evidence from Bostwana », l'intérêt à la veille par rapport à six secteurs de l'environnement a été mesurée en proposant aux questionnés cinq niveaux d'intérêt. L'échelle de mesure de l'intérêt à la veille pour chaque secteur de l'environnement se situe entre (Ce n'est pas intéressant du tout et très intéressant).

En 2000 à Hong kong, Bahman P. Ebrahimi dans son travail « Perceived Strategic Uncertainty and Environmental Scanning Behavior of Hong Kong 
Chinese Executives ", L'intérêt à la veille par rapport à six secteurs de l'environnement a été mesurée en proposant aux questionnés sept (07) niveaux d'intérêt. L'échelle de mesure de l'intérêt à la veille pour chaque secteur de l'environnement se situe entre (intérêt faible et intérêt élevé).

En 2009 à Hong kong, Giri Jogaratnam dans son travail « Environmental Uncertainty and Scanning Behavior: An Assessment of Top-Level Hotel Executives ", Le degré d'interet à la veille par rapport à Six secteurs de l'environnement a été mesurée à l'aide d'une échelle de mesure similaire à celle utilisée par Ebrahimi 2000. Cet instrument de mesure se base sur une échelle de sept (07) points.

\subsection{Le Nombre D'heures Consacrées à La Veille}

Cet item se réfère aux nombre d'heures consacrées par le haut manager à sa pratique de veille par rapport à un secteur de l'environnement. Malgré sa présence au niveau des références théoriques, notre revue de la littérature a montré une quasi absence de cet item au niveau des questionnaires administrés.

En 1993 au Canada, Choo Wei Chun avec son travail intitulé «Environmental scanning: acquisition and use of information by chief executive officers in the canadian telecommunications industry » et lors de son travail avec Auster Eyhel (1993) intitulé «Environmental scanning by CEO's in two Canadian industries », le nombre d'heures consacrées par le hauts manager à sa pratique de veille a été mesuré à l'aide de deux questions directes par rapport aux six (06) secteurs de l'environnement.

- Dans un jour normal de travail, Quel est le nombre d'heures que vous consacré au travail (travail à la maison inclut)?

- Dans un jour normal, Quel est le nombre d'heures que vous consacrez à la pratique de veille ?

\section{Le Mode de Veille}

La revue de la littérature indique également que les hauts managers des entreprises utilisent différents modes de collecte de l'information pour se renseigner sur l'environnement. La distinction entre les différents modes utilisés se fait entre les sources d'information personnelles et impersonnelles et si l'information provient de sources externes ou internes à l'organisation (Culnan, 1983). Les sources personnelles résultent d'un contact humain direct ou par un moyen de communication (Téléphone, mail, ...). Les sources non personnelles sont écrites et comprennent les rapports officiels, les journaux, les résultats des sondages et les informations dégagées par les systèmes informatisés de gestion. 
La littérature indique que les informations personnelles constituent la source d'information la plus commode chez les hauts managers des entreprises, et sont plus compatibles avec les pratiques informelles et irrégulières de la veille stratégique qui caractérisent la grande partie des entreprises (Keegan, 1974; Cité par Choo, 1993). Des recherches relativement récentes concernant le traitement et la gestion de l'information suggèrent que les sources d'informations personnelles sont plus riches que les sources écrites. Les sources d'informations personnelles sont plus riches en terme de contenu et permettent aux hauts managers de détecter des signaux faibles et facilitent ainsi la compréhension lorsque l'incertitude environnementale est élevée.

Une autre classification du mode de veille a été identifiée par (Culnan \& Hambrick, 1983), et se base sur la provenance externe ou interne de l'information. Les sources internes se rapportent aux données, rapports, Procès verbaux des réunions ou discussions avec les gestionnaires internes et les employés au sujet de l'environnement externe. Les sources externes comprennent les rapports des visites, des communications avec les partenaires dans d'autres entreprises, les revues, les journaux, etc.

Considéré comme l'un des instruments de mesure les plus appropriés pour connaître les choix du haut manager par rapport aux sources d'information utilisées lors de sa pratique de veille, «le mode de veille » a été opérationnalisé de différentes manières par les auteurs.

Concernant notre étude qualitative et lors de nos entretiens semi-directifs avec les hauts managers de l'industrie pharmaceutique marocaine, on a pu constater chez nos interlocuteurs une acceptation de cette classification des sources d'information et par conséquent une bonne assimilation des items liés au mode de veille stratégique "Source interne »; "Source externe »; « source personnelle » et « Source impersonnelle».

Extraits de verbatim illustrant notre propos:

Une directrice de communication confirme implicitement l'exactitude de notre classification des sources d'information lors de sa pratique de veille : «[...] L'incertitude nous pousse généralement à utiliser des sources personnelles car Elles sont faciles [...] les contacts personnels sont des sources pertinentes qui demandent moins d'effort et faciles [...] c'est tout à fait normal, pour éviter l'incertitude ou le minimiser, les contacts externes, comme source d'information, reste plus claires et facile $[\ldots] \gg$ Interviewé $\mathbf{n}^{\circ} \mathbf{1 3}$.

[...] je m'informe par rapport à la concurrence chez mon réseau de contact que j'entretiens en dehors de la boite et chez la clientèle.....les sources externes sont facilement accessibles [...] les informations les plus utiles sont les sources personnelles [...] Les sources internes servent à développer la fiabilité d'une source externe» Interviewé $\boldsymbol{n}^{\circ} 8$. 
En 1988 aux Etats Unis d'Amérique, Daft et d'autres avec leur travail intitulé "Chief executive scanning, environmental characteristics, and company performance: an empirical study », le mode de veille du haut manager a été déterminé en proposant aux questionnés les quatre (04) sources suivantes :

Lors de votre pratique de veille, quelles sont les sources d'informations qui vous paraissent les plus utiles?

- Les sources écrites externes (Journaux, Magasine spécialisé, service d'information, etc...)

- Les sources écrites internes (Etudes et rapports réalisés en interne, base de données internes, etc...)

- Les contacts personnels externes (Associés, Fonctionnaires, Clients, Visites, etc..)

- Les contacts personnels internes (Subalternes, Equipe de travail, membre du conseil d'administrations, etc....)

En 2009 à Hong kong, Giri Jogaratnam dans son travail « Environmental Uncertainty and Scanning Behavior: An Assessment of Top-Level Hotel Executives ", Le mode de veille a été mesuré à l'aide d'une échelle de mesure similaire à celle utilisée par Choo et Auster 1993.

\section{L'incertitude Perçue de L'environnement}

H. Mintzberg (1973) fait la distinction entre la stratégie réalisée et la stratégie voulue et retient l'environnement de l'entreprise comme facteur de contingence susceptible d'influencer la stratégie de l'entreprise. Pour Ansoff, l'introduction du facteur environnemental à coté de celui de l'aptitude au management est à l'origine de la remise en question de la planification stratégique et de sa substitution par le concept de management stratégique. Or, l'environnement par rapport auquel la décision est prise n'est pas objectif, c'est un environnement perçu, un ensemble de perceptions complexes, évolutives, souvent contradictoires ; c'est un artefact qui n'est qu'une représentation partielle et déformée de l'environnement réel (Porter \& Millar, 1985) (Cité par Bergeron, F et al., 1988).

Généralement, l'environnement externe peut être classifié en deux catégories : le micro environnement qui a un impact direct et à court terme sur l'activité de l'entreprise et le macro environnement avec des impacts indirects et à long terme (Xue Zhang, Shaheen Majid \& Schubert Foo, 2011).

Duncan (1972) définit l'environnement comme « Les facteurs sociaux et physiques pertinents qui se trouvent en dehors des frontières de l'entreprise et qui sont pris en considération lors de la prise de décision ». Thiétart (1984), définit l'environnement comme : « un ensemble d'acteurs ayant des objectifs individualisés, poursuivant des buts, parfois contradictoires, 
pouvant aider à la réalisation de la stratégie ou bien au contraire, y porter un préjudice irrémédiable ».

Concernant l'incertitude environnementale, ce concept a longtemps été vu comme un concept central dans la littérature de la théorie d'organisation, particulièrement dans des théories cherchant à expliquer la nature de la relation entre les organisations et leurs environnements (Jennifer L. Harrison, 2003). C'est une notion tout à fait relative, contingente par rapport à l'entreprise, qui peut correspondre aussi bien à un manque qu'à une surabondance d'information associés à une situation ambigüe où le décideur se trouve incapable de prévoir les changements de l'environnement. Les études pionnières essayant de mesurer l'incertitude environnementale se subdivisent généralement en deux. Celles qui cherchent à la mesurer objectivement et d'autres qui tentent à mesurer l'incertitude de l'environnement perçue.

Selon Duncan (1972), le concept d'incertitude a été défini de différentes façons dans la littérature. En effet certains théoriciens, insistant sur l'aspect mathématique, et définissent l'incertitude d'un environnement comme le nombre des résultats possibles que pourraient avoir l'événement. D'autres théoriciens de la décision définissent l'incertitude comme la situation où les probabilités des résultats des événements sont inconnues contrairement aux situations de risque où chaque résultat a une probabilité connue.

La grande partie des travaux ayant cherché à expliquer la corrélation entre l'incertitude de l'environnement et l'utilisation de la veille stratégique opte pour la notion de l'incertitude stratégique perçue de l'environnement. Par cette dernière, Daft et al. (1988), entendent l'absence d'information concernant les organisations, les activités et les événements dans l'environnement. Milliken, F.J. (1987) a défini l'incertitude stratégique perçue comme "l'incapacité perçue de l'individu de prévoir quelque chose d'une manière exacte ». Selon cet auteur lorsque le terme " environnement » est attaché au terme incertitude, cela suggère que la source de l'incertitude est l'environnement externe de l'organisation.

Trois dimensions environnementales influencent l'incertitude perçue, soit la complexité perçue, la variabilité perçue et l'importance perçue (Duncan, 1972; Daft, 1988).

La complexité se réfère à l'hétérogénéité des événements extérieurs qui se rapportent à l'organisation, tandis que la variabilité se réfère à l'augmentation $\mathrm{du}$ rythme $\mathrm{du}$ changement dans les secteurs de l'environnement (Duncan, 1972).

Concernant notre étude exploratoire qualitative et lors de nos entretiens semi-directifs avec les hauts managers de l'industrie pharmaceutique marocaine, on a pu constater une acceptation conditionnée des items. En effet, la compréhension des questions liées aux concepts de «complexité », 
de «variabilité » et «d'importance » suppose certaines explications qui accompagnent l'item.

Extraits de verbatim illustrant notre propos :

Un autre directeur de produit s'exprime par rapport aux sources d'incertitude: "[...] je pense qu'à l'heure actuelle, et avec le développement des NTIC, tous les secteurs de l'environnement sont changeants, mais la concurrence est le secteur le plus rapide en terme de changement à un niveau qu'elle commence à créer $d u$ besoin au client [...] bien sûr, l'environnement macro est changeant mais moi je vous parle des secteurs les plus importants [...] l'incertitude émane beaucoup plus du micro environnement» Interviewé $\mathbf{n}^{\circ} 12$.

Un autre haut manager (Directeur de produit) affirme l'importance des changements émanant généralement du micro environnement et plus particulièrement de la concurrence : " [...] Bien évidement, mon principal souci c'est le concurrent [...] ce qui nous importe le plus c'est tout ce qui est concurrence, fournisseurs, clientèle [...] ce qui crée, pour nous, plus d'incertitude c'est notre micro environnement» Interviewé nº.

\subsection{La Complexité Perçue de L'environnement}

La complexité se réfère à l'hétérogénéité et la diversité des événements extérieurs qui impactent l'organisation, ainsi que le nombre d'intervenants dans un secteur environnemental (Humbrick, 1983). Une grande part des chercheurs ont essayé d'opérationnaliser cette dimension via une question directe par rapport au degré de complexité perçu en proposant aux questionnés une échelle de cinq (05) point allant de trop faible jusqu'à trop élevé.

En 1993 au Canada, Choo Wei Chun avec son travail intitulé «Environmental scanning: acquisition and use of information by chief executive officers in the canadian telecommunications industry », et lors de son travail avec Auster Eyhel (1993) intitulé «Environmental scanning by CEO's in two Canadian industries », la complexité perçue de l'environnement a été mesuré via l'item suivant:

Quel est le degré de complexité (hétérogénéité et diversité des évènements) de chaque secteur de l'environnement ?

Tableau $\mathrm{N}^{\circ} 4$. Instrument de mesure de la complexité percue de l'environnement

\begin{tabular}{|c|c|c|c|c|c|}
\multicolumn{1}{c|}{} & Bas & $\begin{array}{c}\text { Assez } \\
\text { bas }\end{array}$ & Moyen & Assez élevé & Elevé \\
\hline Secteur de la concurrence & 1 & 2 & 3 & 4 & 5 \\
\hline Secteur de la clientèle & 1 & 2 & 3 & 4 & 5 \\
\hline Environnement technologique & 1 & 2 & 3 & 4 & 5 \\
\hline l'environnement juridique & 1 & 2 & 3 & 4 & 5 \\
\hline l'environnement économique & 1 & 2 & 3 & 4 & 5 \\
\hline l'environnement socioculturel & 1 & 2 & 3 & 4 & 5 \\
\hline
\end{tabular}

Source : Choo (1993) 
En 2000 à Hong kong, Bahman P. Ebrahimi dans son travail « Perceived Strategic Uncertainty and Environmental Scanning Behavior of Hong Kong Chinese Executives " et en 2009 à Hong kong, Giri Jogaratnam dans son travail « Environmental Uncertainty and Scanning Behavior: An Assessment of Top-Level Hotel Executives ", La complexité perçue de l'environnement a été mesurée à l'aide d'un instrument de mesure similaire à celui utilisé par Choo et Auster 1993 sauf que l'échelle utilisée est de sept points au lieu de cinq.

\subsection{La Variabilité ou Le Degré de Changement}

La variabilité se réfère au degré de changement et à la dynamique des événements extérieurs impactent l'organisation (Humbrick, 1983). Une grande part des chercheurs ont essayé d'opérationnaliser cette dimension via une question directe par rapport au degré de variabilité perçu dans chaque secteur de l'environnement.

En 1993 au Canada, Choo Wei Chun avec son travail intitulé «Environmental scanning: acquisition and use of information by chief executive officers in the canadian telecommunications industry », et lors de son travail avec Auster Eyhel (1993) intitulé «Environmental scanning by CEO's in two Canadian industries ", la variabilité perçue de l'environnement a été mesuré via l'item suivant :

- Quel est le degré de changement dans chaque secteur de l'environnement?

Tableau N N$^{\circ}$. Instrument de mesure de la Variabilité percue de l'environnement

\begin{tabular}{|c|c|c|c|c|c|}
\hline \multicolumn{1}{c|}{} & Bas & Assez bas & Moyen & Assez élevé & Elevé \\
\hline Secteur de la concurrence & 1 & 2 & 3 & 4 & 5 \\
\hline Secteur de la clientèle & 1 & 2 & 3 & 4 & 5 \\
\hline Environnement technologique & 1 & 2 & 3 & 4 & 5 \\
\hline l'environnement juridique & 1 & 2 & 3 & 4 & 5 \\
\hline l'environnement économique & 1 & 2 & 3 & 4 & 5 \\
\hline
\end{tabular}

Source : Choo (1993)

En 1997, Detelin s. elenkov dans son travail «strategic uncertainty and environmental scanning: the case for institutional influences on scanning behavior", La variabilité perçue de l'environnement a été mesurée à l'aide d'un instrument de mesure similaire à celui utilisé par Choo et Auster 1993.

En 2000 à Hong kong, Bahman P. Ebrahimi dans son travail «Perceived Strategic Uncertainty and Environmental Scanning Behavior of Hong Kong Chinese Executives " et en 2009 à Hong kong, Giri Jogaratnam dans son travail « Environmental Uncertainty and Scanning Behavior: An Assessment of Top-Level Hotel Executives ", La variabilité perçue de l'environnement a 
été mesurée à l'aide d'un instrument de mesure similaire à celui utilisé par Choo et Auster 1993 sauf que l'échelle utilisée est de sept points au lieu de cinq.

\subsection{L'importance perçue de l'environnement}

Cette dimension se réfère au degré d'importance accordé par le haut manager aux changements et événements liés à un secteur environnemental. Une grande part des chercheurs ont essayé d'opérationnaliser cette dimension via une question directe par rapport au degré d'importance de chaque secteur de l'environnement. Selon les chercheurs, cette dimension constitue le principal déterminant de la perception de l'incertitude stratégique chez le haut manager (Pfeffer \& Salancik, 1978; Aaker, 1983). L'information provenant d'un secteur environnemental important peut apporter un avantage stratégique (Dutton \& Freedman, 1984). L'importance et l'incertitude de l'environnent créent ce qu'on appelle l'incertitude stratégique de l'environnement.

En 1988 aux Etats Unis d'Amérique, Daft et d'autres avec leur travail intitulé "Chief executive scanning, environmental characteristics, and company performance: an empirical study », Le degré d'importance d'un secteur environnemental a été mesuré à l'aide d'une échelle de mesure similaire à celle utilisée par Hambrick en 1982 et Culnan 1984 et validée par Farh et d'autres en 1984. Cette mesure est basée sur une échelle de cinq (05) points allant d'une importance très faible à une importance trop élevée.

En 1993 au Canada, Choo Wei Chun avec son travail intitulé «Environmental scanning: acquisition and use of information by chief executive officers in the canadian telecommunications industry », et lors de son travail avec Auster Eyhel (1993) intitulé «Environmental scanning by CEO's in two Canadian industries ", l'importance perçue d'un secteur de l'environnement a été mesurée via l'item suivant :

Comment vous jugez le degré d'importance des événements et des changements dans chaque secteur de l'environnement ? 
Tableau Nº6. Instrument de mesure de l'importance percue de l'environnement

\begin{tabular}{|c|c|c|c|c|c|}
\hline $\begin{array}{c}\text { La concurrence } \\
\begin{array}{c}\text { (Les structures produisant des produits } \\
\text { qui peuvent substituer votre produit) }\end{array}\end{array}$ & $\begin{array}{c}\text { Ce n'est } \\
\text { pas } \\
\text { important }\end{array}$ & $\begin{array}{c}\text { Assez } \\
\text { non } \\
\text { importa } \\
\text { nt }\end{array}$ & $\begin{array}{c}\text { Moyennem } \\
\text { ent } \\
\text { important }\end{array}$ & $\begin{array}{c}\text { Assez } \\
\text { import } \\
\text { ant }\end{array}$ & $\begin{array}{c}\text { Très } \\
\text { importa } \\
\text { nt }\end{array}$ \\
\hline $\begin{array}{c}\text { La clientèle } \\
\text { (Structures ou particuliers qui achètent } \\
\text { votre produit y compris les } \\
\text { distributeurs et les revendeurs) }\end{array}$ & 1 & 2 & 3 & 4 & 5 \\
\hline $\begin{array}{c}\text { Environnement technologique } \\
\text { (Le développement de nouveaux } \\
\text { produits techniques, innovations, } \\
\text { Recherche et développement liés à } \\
\text { votre domaine d'activité) }\end{array}$ & 1 & 2 & 3 & 4 & 5 \\
\hline $\begin{array}{c}\text { l'environnement juridique } \\
\text { (le changement de la réglementation } \\
\text { en vigueur, de la représentation } \\
\text { politique) }\end{array}$ & 1 & 2 & 3 & 4 & 5 \\
\hline $\begin{array}{c}\text { l'environnement économique } \\
\text { (les facteurs économiques comme le } \\
\text { marché boursier, inflation, les taux } \\
\text { d'intérêts, le chômage, la croissance } \\
\text { économique) }\end{array}$ & 1 & 2 & 3 & 4 & 5 \\
\hline $\begin{array}{c}\text { l'environnement socioculturel } \\
\text { (Tendances démographiques, Attitudes } \\
\text { sociales) }\end{array}$ & 1 & 2 & 3 & 5 & 5 \\
\hline
\end{tabular}

Source : Choo (1993)

\section{L'accessibilité Prçue D'une Source D'information}

L'accessibilité a été définie comme : "le degré avec lequel un chercheur de l'information perçoit la facilité d'obtenir, d'approcher ou d'entrer en contact avec un canal d'information ciblé » (Allen, 1977, p182, cité par Choo, 1993, p 92). Cette perception de l'accessibilité se fait sans une prise de considération de la fiabilité ou de la qualité de l'information attendue.

En sciences économiques et de management de l'information et de documentation, l'accessibilité est généralement définie en termes de coûts "physiques" d'utilisation, en particulier la distance physique entre la source de l'information et son utilisateur. Culnan (1985) définit l'accessibilité perçue comme le «niveau attendu de l'effort requis pour utiliser une source d'information particulière ».

Concernant les dimensions de l'accessibilité perçue de l'information, O'Reilly (1982) propose trois questions pour mesurer l'accessibilité perçue de l'information. Ces questions concernent le temps, la dépense et la difficulté 
d'obtenir des informations de la source. Par rapport à notre problématique de recherche, nous avons opté pour «le temps et les efforts nécessaires pour aborder, contacter ou de localiser la source » et « la facilité d'accès à la source de l'information » comme les deux dimensions de l'accessibilité perçue de la source. Ces deux dimensions sont opérationnalisées sur le terrain à travers deux questions adaptées des définitions théoriques d'Allen (1977) et Culnan (1985), et les questions élaborées par O'Reilly (1982).

Concernant notre étude exploratoire qualitative et lors de nos entretiens semi-directifs avec les hauts managers de l'industrie pharmaceutique marocaine, on a pu constater que nos interviewés n'arrivent plus à faire la distinction entre «l'effort fourni pour accéder à une source » et « la facilité d'obtention d'une information à partir d'une source ». La confusion entre les deux items a dominé les entretiens et la majorité des hauts managers a jugé les deux questions de semblables.

Extraits de verbatim illustrant notre propos:

Une directrice de communication confirme l'accessibilité comme critère de choix d'une source d'information et fais la confusion entre la facilité d'accès et la facilité d'obtention d'une information à partir d'une source: " [...] Une source d'information facile et exacte est Bâ certainement c'est la préférée [...] L'incertitude nous pousse généralement à utiliser des sources personnelles car Elles sont faciles et accessible [...] les contacts personnels sont des sources pertinentes qui demandent moins d'effort et faciles [...] pas souvent mais l'accessibilité est un facteur déterminant pour le choix d'une source [...] c'est tout à fait normal, pour éviter l'incertitude ou le minimiser, les contacts externes, comme source d'information, reste plus claires et facile[...] dans le monde des affaires, on ne fait plus confiance aux gens, mais les banquiers et les amis les plus proches restent le choix dont je fais plus confiance » Interviewé nº13.

En 1993 au Canada, Choo Wei Chun avec son travail intitulé «Environmental scanning: acquisition and use of information by chief executive officers in the canadian telecommunications industry », et lors de son travail avec Auster Eyhel (1993) intitulé «Environmental scanning by CEO's in two Canadian industries ", l'accessibilité d'une source d'information en terme de temps et d'effort a été mesurée via l'item suivant :

- Combien de temps et d'efforts sont nécessaires pour approcher, contacter ou de localiser chaque source d'information? 
Tableau N7. Instrument de mesure de l'accessibilité percue d'une source d'information en termes de temps et d'effort

\begin{tabular}{|c|c|c|c|c|c|c|}
\hline $\begin{array}{c}\text { Sources } \\
\text { d'information }\end{array}$ & Composantes & $\begin{array}{c}\text { Vraiment } \\
\text { beaucoup }\end{array}$ & Beaucoup & Considérable & Peu & $\begin{array}{c}\text { Très } \\
\text { peu }\end{array}$ \\
\hline \multirow{4}{*}{$\begin{array}{c}\text { Sources } \\
\text { personnelles } \\
\text { externes }\end{array}$} & Clients & 1 & 2 & 3 & 4 & 5 \\
\cline { 2 - 7 } & $\begin{array}{c}\text { Concurrents } \\
\text { professionnels }\end{array}$ & 1 & 2 & 3 & 4 & 5 \\
\cline { 2 - 7 } & Collaborateurs & 1 & 2 & 3 & 4 & 5 \\
\cline { 2 - 7 } & $\begin{array}{c}\text { Fonctionnaires, représentants } \\
\text { du gouvernement }\end{array}$ & 1 & 2 & 3 & 4 & 5 \\
\hline \multirow{4}{*}{$\begin{array}{c}\text { Sources } \\
\text { externes non } \\
\text { personnelles }\end{array}$} & Journaux et Périodiques & 1 & 2 & 3 & 4 & 5 \\
\cline { 2 - 7 } & Rapports du gouvernement & 1 & 2 & 3 & 4 & 5 \\
\cline { 2 - 7 } & Média (Radio, TV, etc..) & 1 & 2 & 3 & 4 & 5 \\
\cline { 2 - 7 } & Associations industrielles & 1 & 2 & 3 & 4 & 5 \\
\hline \multirow{2}{*}{$\begin{array}{c}\text { Sources } \\
\text { personnelles } \\
\text { internes }\end{array}$} & $\begin{array}{c}\text { Supérieurs et membres du } \\
\text { conseil d'administration }\end{array}$ & 1 & 2 & 3 & 4 & 5 \\
\cline { 2 - 7 } & Cadres Subalternes & 1 & 2 & 3 & 4 & 5 \\
\hline \multirow{3}{*}{$\begin{array}{c}\text { Sources } \\
\text { internes non } \\
\text { personnelles }\end{array}$} & Equipe subalterne & 1 & 2 & 3 & 4 & 5 \\
\cline { 2 - 7 } & Notes de service et circulaires & 1 & 2 & 3 & 4 & 5 \\
\cline { 2 - 7 } & Etudes et Rapports internes & 1 & 2 & 3 & 4 & 5 \\
\cline { 2 - 7 } & Bibliothèque de l'entreprise & 1 & 2 & 3 & 4 & 5 \\
\hline \multirow{2}{*}{$\begin{array}{c}\text { Service d'information } \\
\text { électronique }\end{array}$} & 1 & 2 & 3 & 4 & 5 \\
\hline
\end{tabular}

Source : Choo (1993)

Dans le même sens, Choo a essayé de mesurer l'accessibilité d'une source en termes de facilité d'accès via la question suivante:

- Après avoir contacté ou localisé la source, est-il facile d'obtenir les informations souhaitées provenant de cette source?

Tableau $N^{\circ} 8$. Instrument de mesure de l'accessibilité percue d'une source d'information en terme de facilité

\begin{tabular}{|c|c|c|c|c|c|c|}
\hline $\begin{array}{c}\text { Sources } \\
\text { d'information }\end{array}$ & Composantes & $\begin{array}{c}\text { Très } \\
\text { difficile }\end{array}$ & $\begin{array}{c}\text { Peu } \\
\text { Difficile }\end{array}$ & $\begin{array}{c}\text { Ni facile ni } \\
\text { difficile }\end{array}$ & $\begin{array}{c}\text { Peu } \\
\text { facile }\end{array}$ & $\begin{array}{c}\text { Très } \\
\text { facile }\end{array}$ \\
\hline \multirow{4}{*}{$\begin{array}{c}\text { Sources } \\
\text { personnelles } \\
\text { externes }\end{array}$} & Clients & 1 & 2 & 3 & 4 & 5 \\
\cline { 2 - 7 } & Concurrents & 1 & 2 & 3 & 4 & 5 \\
\cline { 2 - 7 } & $\begin{array}{c}\text { Hommes d'affaires et } \\
\text { professionnels }\end{array}$ & 1 & 2 & 3 & 4 & 5 \\
\cline { 2 - 7 } & $\begin{array}{c}\text { Collaborateurs } \\
\text { Fonctionnaires, représentants du } \\
\text { gouvernement }\end{array}$ & 1 & 2 & 3 & 4 & 5 \\
\hline \multirow{3}{*}{$\begin{array}{c}\text { Sources } \\
\text { pxternes non } \\
\text { personnelles }\end{array}$} & Journaux et Périodiques & 1 & 2 & 3 & 4 & 5 \\
\cline { 2 - 7 } & Rapports du gouvernement & 1 & 2 & 3 & 4 & 5 \\
\cline { 2 - 7 } & Média (Radio, TV, etc..) & 1 & 2 & 3 & 4 & 5 \\
\cline { 2 - 7 } $\begin{array}{c}\text { Sources } \\
\text { personnelles } \\
\text { internes }\end{array}$ & Associations industrielles & 1 & 2 & 3 & 4 & 5 \\
\cline { 2 - 7 } & Colloques et visites & 1 & 2 & 3 & 4 & 5 \\
\cline { 2 - 7 } & Supérieurs et membres du conseil & 1 & 2 & 3 & 4 & 5 \\
\cline { 2 - 7 } & Cadministration & 1 & 2 & 3 & 4 & 5 \\
\hline
\end{tabular}




\begin{tabular}{|c|c|c|c|c|c|c|}
\multirow{4}{*}{$\begin{array}{c}\text { Sources } \\
\text { internes non } \\
\text { personnelles }\end{array}$} & Notes de dervice et circulaires & 1 & 2 & 3 & 4 & 5 \\
\cline { 2 - 7 } & Etudes et Rapports internes & 1 & 2 & 3 & 4 & 5 \\
\cline { 2 - 7 } & Bibliothèque de l'entreprise & 1 & 2 & 3 & 4 & 5 \\
\cline { 2 - 7 } & $\begin{array}{c}\text { Service d'information } \\
\text { électronique }\end{array}$ & 1 & 2 & 3 & 4 & 5 \\
\hline
\end{tabular}

Source : Choo (1993)

\section{La Qualité Perçue D'une Source D'information}

Basé sur une revue de la littérature relative aux caractéristiques d'une source d'information, Zmud (1978) a identifié les trois dimensions suivantes, soit la quantité qui est mesurée par des adjectifs tels que "suffisante et complète ", la fiabilité qui est mesurée par le degré de précision et d'exactitude caractérisant une information, et la qualité de sa forme de présentation mesurée par la lisibilité et la clarté. Pour valider empiriquement ces dimensions, Zmud a obtenu quatre niveaux pour mesurer la qualité d'une information : un premier niveau qui se base sur la mesure de la pertinence de l'information; un deuxième niveau qui se base sur la mesure des composants de la pertinence à savoir l'exactitude, la quantité, la fiabilité ; un troisième niveau lié à la mesure de la qualité de la forme de présentation de l'information et de sa lisibilité ; et le quatrième niveau lié à la qualité du sens de l'information en terme de ses caractères raisonnable et logique.

Le concept de pertinence est fondamental dans le développement des sciences de l'information. Saracevic (1970) définit la pertinence comme « une mesure de l'utilité d'une information ». Pour Eisenberg et Schamber (1991) "la pertinence de l'information est une mesure de l'utilité existant entre un document et une question jugée importante par un chercheur». Dans son étude comparant l'impact de la qualité d'une source d'information sur l'utilisation des sources d'information par les décideurs, O'Reilly (1982) a précisé que la pertinence est une dimension principale de la qualité perçue d'une source d'information.

De son coté, Taylor suggère la notion de la fiabilité comme l'une des dimensions principale qui donnent de la valeur à une source. Il définit la "fiabilité » comme : "la confiance qu'un utilisateur dispose par rapport à la cohérence, la performance de la qualité d'un système et ses résultats au fil du temps » (Taylor, 1986; cité par Choo, 1993, p 94). D'après une étude menée par Nilan, Peek et Snyder (1988), et qui cherchait à étudier les critères d'évaluation d'une source d'information, ils ont identifié la fiabilité comme la seconde dimension de la qualité d'une source d'information.

Concernant notre étude exploratoire qualitative et lors de nos entretiens semi-directifs avec les hauts managers de l'industrie pharmaceutique marocaine, on a pu constater une parfaite acceptation des items liés à la fiabilité dans le sens du degré de confiance par rapport à une source d'information et à la pertinence dans le sens de l'utilité d'une source. 
Extraits de verbatim illustrant notre propos:

Une directrice de communication confirme la fiabilité et la pertinence comme critères de choix d'une source d'information : " [...] le fait de préférer une source est une question de confiance et du temps .....Les sources externes sont facilement accessibles [...] les informations les plus utiles sont les sources personnelles [...] Les sources internes servent à développer la fiabilité d'une source externe» Interviewé n8.

" [...] pour moi, les deux sources (externes et internes) sont sine qua non et complémentaires [...] les sources formelles sont plus préférées car elles sont exactes [...] les sources les plus utiles sont les conversations téléphoniques avec les amis [...] les sources les plus fiables sont les sources internes alors que les sources externes restent toujours des sources à tester» Interviewé $\mathbf{n}^{\circ} \mathbf{1}$.

Pour mesurer la qualité perçue de la source, nous optons dans le cadre de ce travail pour les deux dimensions suivantes : soit « la pertinence » et « la fiabilité ». Ces deux dimensions seront opérationnalisées sur le terrain en se basant sur les deux questions suivantes:

- Quel est le degré de pertinence de l'information provenant de chaque source de l'environnement?

Les Informations pertinentes sont des informations qui sont jugées nécessaires et utiles par rapport aux objectifs et les activités de l'entreprise.

- Quel est le degré de fiabilité de l'information provenant de chaque source de l'environnement?

Les Informations fiables sont des informations qui sont jugées sûres et dont l'utilisateur fait confiance.

\section{Le Haut Manager}

Mintzberg (1973) énonce clairement que l'acquisition, la diffusion et l'utilisation de l'information est au cœur du travail du haut manager. Mintzberg a ainsi devisé le travail de manager en dix rôles répartis sur trois niveaux : l'information, le contact avec les personnes et l'action. En dépit des interactions dynamiques qui lient les trois niveaux du rôle du manager au sein de l'entreprise, nous cherchons dans le cadre de notre problématique à éclairer davantage le rôle informationnel du manager.

Le Rôle D'observateur Actif: Le fait d'avoir un rôle de liaison permet au manager d'avoir beaucoup plus d'informations que son équipe. La recherche d'informations est une des clés de la profession de manager. Dans le rôle d'observateur actif, le manager scrute en permanence son entourage à la recherche d'informations.

Le Rôle de Diffuseur D'information: De part son poste, son réseau, et son activité d'observateur actif, le manger dispose de nombreuses 
informations qu'il diffuse en retour à ses équipes, à son réseau ou à ses relations selon les circonstances

Le Rôle de Porte Parole: Le manager a également un rôle de porte parole "officiel" en charge de la communication, aussi bien vers l'extérieur en tant que porte parole de ses équipes, que vers l'intérieur en tant que porte parole de la hiérarchie.

Ces rôles informationnels confirment notre choix du haut manager comme objet d'étude car ce dernier est le mieux positionné au sein de l'entreprise pour l'utilisation de la veille stratégique en tant que pratique informationnelle qui permet plus de compréhension et plus de réactivité par rapport à l'environnement.

\section{CONCLUSION}

Au terme de cette exploration aussi bien de la littérature que du contexte industriel marocain, nous avons pu mettre le doigt sur les instruments de mesure les plus adaptés à notre contexte marocain et les plus objectifs par rapport à notre accessibilité à l'information dans un environnement qui rejette toujours le chercheur académique et dans un climat non sensibilisé à l'apport scientifique.

S'inscrivant dans le cadre d'un travail de thèse et plus particulièrement dans sa phase confirmatoire, ce papier résulte d'un ensemble d'items qui forment notre questionnaire. Des items choisis objectivement et qui vont servir à une opérationnalisation scientifiquement fondée de nos variables de recherche dans le contexte industriel marocain.

\section{References:}

1. Ansoff H. Igor (1975). Managing Strategic Surprise by Response to Weak Signals. California Management Review, Vol. 18 (pp. 21-33).

2. Bahman P. Ebrahimi (2000). Perceived Strategic Uncertainty and Environmental Scanning Behavior of Hong Kong Chinese Executives. University of Denver (p. 11).

3. Bassoul, F. Z. (2010). La pratique de l'intelligence économique dans la PME. FSJES Marrakech, Université CADI AYYAD (p.230).

4. Bayode O. Babatunde \& Adebola O. Adebisi (2012). Strategic Environmental Scanning and Organization Performance in a Competitive Business Environment. Economic Insights, Trends and Challenge, Vol. LXIV (p. 11).

5. Bergeron \& Buteau (1988). Devancer la concurrence par les systèmes d'information. Revue internationale P.M.E, vol. 1, $\mathrm{n}^{\circ} 3-4$, 1988 (pp.295-307).

6. Boulifa, I. (2008). Identification des facteurs critiques de succès pour la mise en place d'un dispositif de veille stratégique. Thèse de 
doctorat, Université de tunis institut superieur de gestion, année Universitaire, (p. 387).

7. Bourjeois, L.J. (1980). Strategy and Environment: A Conceptual Integration. Review Academy of management, Université de Pittsburg (pp. 25-39).

8. Brouard, F. (2000). Que la veille stratégique se lève : Faisons le point sur la terminologie $d u$ concept ». Congrès ASAC-IFSAM Montréal, Québec (p.10).

9. Brouard, F. (2004). Développement d'un outil diagnostique des pratiques Existantes de la veille stratégique Auprès des pme. Thèse présentée à L'université du Québec à Trois-Rivières (p. 240).

10. Chafik, K. \& Hamdoune, A. (2016). Les facteurs influencant l'utilisation de la veille strategique par les hauts managers : essai d'elaboration d'un modele theorique. REMFO (p.23)

11. Chafik, K. \& Hamdoune, A. (2016). Les facteurs influencant l'utilisation de la veille strategique par les hauts managers : essai d'une étude exploratoire qualitative hybride, REMFO (p.20)

12. Chun Wei Choo (1993). Environmental scanning: Acquisition and use of information by chief executive officers In the canadian telecommunications industry. Thèse de doctorat, Faculty of Library and Information Science University of Toronto (pp. 82-282).

13. Culnan J. Mary (1983). Environmental scanning: the effects of task complexity and source accessibility on information gathering behavior. Journal of the decision sciences institute, volume 14 (pp. 194-206).

14. Daft, R. L., Sormunen, J., \& Parks, D. (1988). Chief executive scanning, environmental characteristics, and company performance. Strategic Management Journal (p.18).

15. Daft, R. L. \& Lengel Robert, H. (1986). Organizational Information Requirements, Media Richness and Structural Design. http://dx.doi.org/10.1287/mnsc.32.5.554 Published Online (pp. 554 571).

16. Deprau, A. (2012). Intelligence économique et protection $d u$ patrimoine informationnel : brevets et Prototypes. Sous la direction de M. Xavier ROCHE, Université panthéon-assas (P.341).

17. Desq, S. (2007). La spécificité de la recherche francophone en systèmes d'information. Revue française de gestion, 2007/7 $\mathrm{n}^{\circ} 176$ (pp. 63-79).

18. Detelin S. Elenkov (1997). Strategic uncertainty and environmental scanning: the case for institutional influences on scanning behavior. Strategic Management Journal, Vol. 18 (p.17). 
19. Dhaoui, C. (2008). Les critères de réussite D'un système d'intelligence économique Pour un meilleur pilotage stratégique Proposition d'un modèle d'évaluation de la réussite d'un système d'intelligence économique mersie. Thèse soutenue à l'Universite Nancy 2 Spécialité : Sciences de l'Information et de la Communication (p. 608).

20. Duncan Robert, B. (1972). Characteristics of Organizational Environments and Perceived Environmental Uncertainty. Administrative Science Quarterly Vol.17 (pp.313-327).

21. Eisenberg Michael \& Linda Schamber (1991). On Defining Relevance. Journal of Education for Library and Information Science, Vol. 31, No. 3 (pp. 238-253).

22. François-Xavier de Vaujany (1987). Les grandes approaches théoriques du système d'information. Hermes sciences (p. 239).

23. Hambrick Donald, C. (1982). Environmental scanning and organizational strategy. Management Strategic journal (pp. 159174).

24. Jean-Louis Monino (2012). L'information au coeur de l'intelligence economique strategique. Revue de recherche sur l'innovation (p.34).

25. Jennifer L. Harrison (2003). Perceived environmental uncertainty: validation of a measure from the accounting literature. Australasian Journal of Business \& Social Inquiry (p.16)

26. Jogaratnam, G. \& Kevin, K. F. W. (2009). Environmental Uncertainty and Scanning Behavior: An Assessment of Top-Level Hotel Executives. International Journal of Hospitality \& Tourism Administration (pp.5-25)

27. Josee Audet (2005). La veille stratégique chez les PME de haute technologie: une étude de cas par comparaisons inter-sites. Université du Québec à Trois-Rivières (p.355)

28. Keegan Warren, J. (1974). Multinational Scanning: A Study of the Information Sources Utilized by Headquarters Executives in Multinational Companies. Administrative Science Quarterly Vol. 19, No. 3 (pp. 411-421)

29. Khandwalla N. Pradip (2004). Competencies for Senior Manager Roles. Vikalpa, vol 29, no 4 (p.14)

30. Leaptrott, J. J. \& Michael Mcdonald (2015). Perceived environmental change as a determinant of managerial information seeking behavior. Journal of Management and Marketing Research. (P.19)

31. Lesca Humbert (2004). Théorie et pratique de la veille : quelques retours d'expérience contribuant à L'émergence $d u$ concept D'intelligence stratégique collective. Ecole Supérieure des Affaires de Grenoble, Université P. Mendès France, Grenoble 2. (P. 50). 
32. Lesca Humbert \& Caron Marie-Laurence (2008). Veille strategique : créer une intelligence collective au sein de l'entreprise (p.18).

33. Mermoud, A. (2008). Besoins et pratiques en veille stratégique en Suisse. Haute École de Gestion de Filière Information Documentaire Suisse (P . 223)

34. Mintzberg, H. (1973). A new look at the Chief Executive's Job. Organizational Dynamics, Volume 1, Issue 3 (PP. 21-30)

35. Myburgh, S. (2004). Competitive Intelligence: Bridging Organizational Boundaries, Information Management Journal (pp. 46-55)

36. Narjes Ayari \& Athissingh Ramrajsingh (2008). Mise en place de processus de veille stratégique et concurrentielle dans le domaine de la réassurance : le cas du département de documentation de BEST Ré. Institut d'Etudes Politiques d'Aix-en-Provence - Université AixMarseille III, Paul Cézanne ( p.13)

37. Nishi Kenyu, Schoderbek Charles \& Schoderbek Peter, P. (1982). Scanning the organizational environment: Some empirical results. Human Systems Management review, vol. 3, no. 4( pp. 233-245)

38. Olu Ojo (2008). Appraisal of the impact of environmental scanning on corporate performance in selected Nigerian banks. Department Of Business Studies Covenant University, Ogun State Nigeria. (P. 12)

39. Olukemi O. Sawyerr (1993). Environmental and environmental canning activities of nigerian manufacturing executives: a comparative analysis. Strategic Management Journal, Vol. 14 (p. 14).

40. O'reilly Charles, A. (1978). Variations in Decision Makers' Use of Information Sources: The Impact of Quality and Accessibility of Information. Journal academy of management, vol. 25 no. 4 (pp. 756771)

41. Porter, M.E. \& Millar, V.E. (1985). How information gives you competitive advantage. Harvard business review No85415 (p.06)

42. Rupcic, N. \& Borovac, S. (2013). Business environment scanning: prerequisite for sustainable learning company, https://bib.irb.hr/datoteka/592302 (p. 11)

43. Salles, M. (2006). Stratégies des PME et intelligence économique. Une méthode d'analyse du besoin. Éditions Économica, Paris, 2ème édition, septembre. (p.204)

44. Sidney E. Harris \& Joseph L. Katz (1991). Organizational Performance and Information Technology Investment Intensity in the Insurance Industry. Published Online: August 1 (pp. 263 - 295)

45. Souad Kamoun-Chouk (2005). Veille Anticipative Stratégique : Processus d'Attention à l'Environnement Application à des PMI 
tunisiennes. CERAG CNRS UMR 5820/ ED 275/UPMF Grenoble France (p. 420)

46. Slim, A. (2012). Veille strategique et performance des petites et moyennes entreprises exportatrices tunisiennes. Université de Lyon 3 institut d'administration des entreprises (IAE) (p.23)

47. Thepaut, Y. (2006). Le concept d'information dans l'analyse économique contemporaine. La Revue Hermès, ${ }^{\circ}$ 44, (pp. 161-168).

48. Thietart, R.A. \& Forgues, B. (2006). La dialectique de l'ordre et du chaos dans les organisations », Revue française de gestion (pp.4766)

49. Zelaelem T. Temtime (2001). Environmental scanning behavior of small and medium firms in Developing economies: Evidence from Bostwana. Pakistan Journal of applied sciences (pp. 1-7)

50. Zmud W. Robert (1978). An Empirical Investigation Of The Dimensionality Of The Concept Of Information . Decision Sciences, Vol 9 (pp. 187-195) 